\title{
Design and Implementation of a Class Constant Frequency Wind Power Generator
}

\author{
Yande Lin \\ School of Automation \\ Shenyang Institute of Engineering \\ Shenyang, China \\ e-mail: yandan91@163.com
}

\author{
Xianguo Li \\ Branch of Chaoyang Power Supply \\ State Grid Liaoning Electric Power Supply Co. Ltd \\ Chaoyang, China \\ e-mail: xianguo1122@126.com
}

\begin{abstract}
As a kind of clean and renewable energy, wind power has many advantages such as a large resource reserves, renewable, no pollution emissions and not flooded, covers less area, short construction period, flexible and so on. Its unique advantages make it stand out in many new energy is becoming a hot spot of attention and research.In this letter, firstly we study the optimal power flow calculation methods of wind power, and consider oscillation problems caused by the constraints based on two models, to achieve the safety of the power system optimal operation of the economy. Secondly, the core technology of wind power is the design and implementation of wind turbine control system. This article uses the doubly-fed motor winding type, designed and implemented a variable speed constant frequency system which contained the experimental mill, The main loop of the ac excitation system, DSP controller, Signal detection circuit, remote grid control circuit and Protection circuit.
\end{abstract}

Keywords-Optimal power flow calculation; Super curve; Wind power generation; Constant frequency system; Experiment mill

\section{INTRODUCTION}

As the world energy crisis and environment problem increasingly prominent, such as "the development and utilization of renewable energy, reducing emissions of greenhouse gases, protecting the environment" has become the consensus of the world's governments. Clean, efficient and renewable energy become the mainstream of energy production and consumption. Wind power has an advantage of large resource reserves, renewable, no pollution emissions and not flood, covering a less area, short construction period, flexible scale, such unique advantages make it stand out in lots of new energy and become a hot spot of attention and research. Since 1990, wind power generation technology obtained a rapid development, the global wind power installed capacity of average annual increases more than $20 \%$. According to the European wind energy association and green-peace signed 《the wind 12》, the global wind power installed capacity in 2020 will reach $1200 \mathrm{GW}$, wind power will account for $12 \%$ of the world's total capacity. Thus, wind power is no longer the dispensable supplement energy, as one of the most commercial prospects for the development of new industry, it has become the indispensable part to solve world's energy problems. The process of wind power generation is to change wind energy into electricity by the process of mechanical energy. Wind generator is the core of the system which is responsible for converting mechanical energy to electrical energy, its status directly affects the performance of the whole system. Therefore, how to realize a high reliability, high efficiency control system suitable for the wind power conversion is the research pot of wind power technology, in this article, we will design and implement a constant frequency system.

\section{THE OPTIMAL POWER FLOW CALCULATION CONTAINING WIND POWER}

In modern power system, there are a lot of problems involving safety and economic optimization operation can be summed up in solving optimization trend. Power flow transmission capacity constraint is an important constraint in optimization of tide problem, in some cases it even become bottleneck constraints of system operation security and the economy. Therefore, the optimization method of research trends of wind power control problem is one of the better direction.

1 Two main types of optimization flow control model based on the wind

1) Global optimization control model based on wind power

2) Custom power transmission mode

Contain optimized trend of wind power control function can be unified with the following formula:

$$
\begin{aligned}
& \min f(x, u) \\
& \left\{\begin{array}{l}
g(x, u) \\
h^{\min } \leq h(x, u) \leq h^{\max }
\end{array}\right.
\end{aligned}
$$

From the above analysis, when using the equivalent power injection of wind model, wind power control function of electric power system optimization equation (including equality constraint equation, equation of inequality constraints and the equation of the objective function, etc.) can be expressed into two parts, one part is the same as regardless of the wind power system optimization equation form; Another part is the expression for the additional power of the wind. With unified expression formula is as follows:

$$
\left\{\begin{array}{l}
f(x, u)=f_{1}\left(x, u_{1}\right)+f_{2}(\mathrm{VS}) \\
g(x, u)=g_{1}\left(x, u_{1}\right)+g_{2}(\mathrm{VS}) \\
(x, u)=h_{1}\left(x, u_{1}\right)+h_{2}(\mathrm{VS})
\end{array}\right.
$$


2 Trend oscillation problems caused by the constraints

Let us suppose that all the inequality constraints equation limit the variables in a bounded area, all current equality constraint equation in the variable space constitute a super curve, the intersection of both constitute the feasible domain of solution vectors, as shown in the rectangular area in Fig .1 and Fig .2

When the initial starting point in the bounded region near the border (1) initial state, the algorithm would make it to the optimal solution under no constraint, which is possible to meet the boundary of the bounded area. But the current solution could not meet the trend of the constraints, if it is not on the super curve. In order to make the final solution on trend curve solutions, algorithm will tend to be oscillated near the border area. If we from a far from bounded area boundary is the initial starting point (2) the initial state, the algorithm will realize the trend of the constraint, and convergence at some point in the region and trend curve, and after finite iterations to reach the optimal solution.

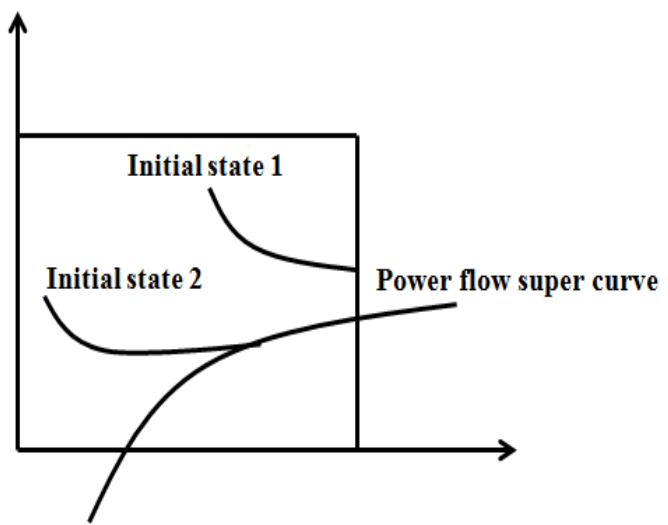

Figure 1. The influence of initial state on the convergence performance

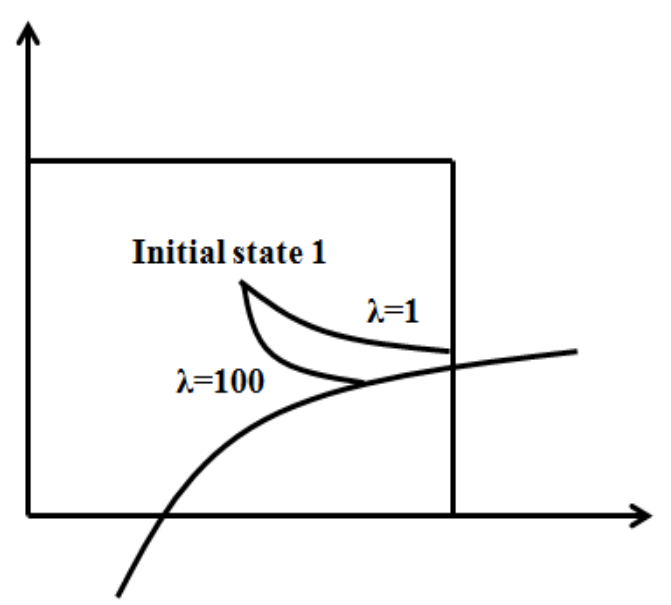

Figure 2. Multiplier effect on the performance of convergence trend of constraints

In addition to consider the voltage characteristic of wind farm of continuous trend of wind power and wind power system simulation, the particularity of wind power generation growth also should be considered. Under the condition of invariable installed in wind farms, the wind power active power depends on the growth of wind resources, wind farm reactive power at the same time have a corresponding change. Because the wind has rendered meritorious service, the characteristics of reactive power, so the continuous trend in the simulation study of wind farm and the access point voltage change of the wind power system and its stability analysis is of great significance.

Here is based on the basic principle of optimal power flow and combined with the advantages of the conventional calculation, introduced the static power of the wind farm voltage characteristic, thus completed wind power system and wind tide simulation and accurately calculate the static voltage stability limit wind farm.

\section{DESIGN AND IMPLEMENTATION}

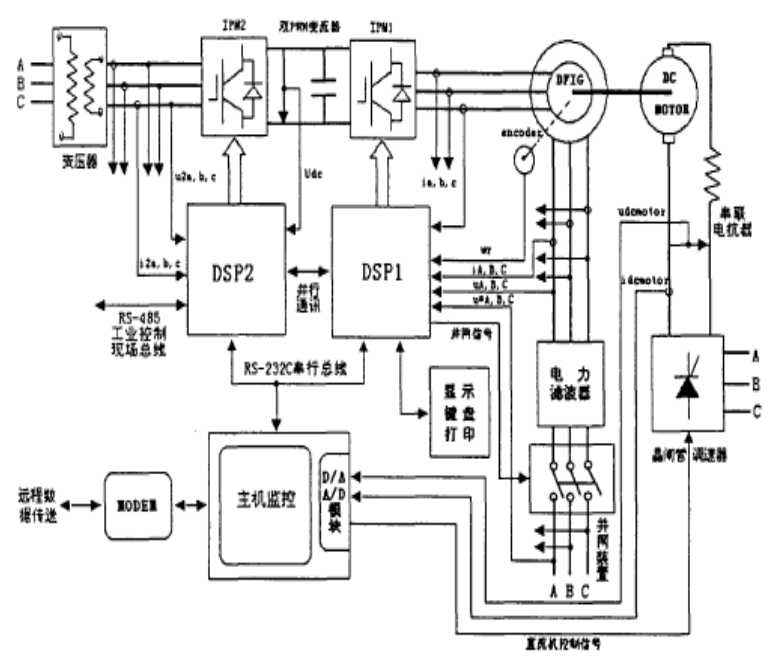

Figure 3. The control block diagram of AC excitation variable speed constant frequency wind power generation system.

The system diagram is shown in Fig .3. The experimental mill is a wire-wound doubly-fed motor drive by a dc motor. The excitation source of generator rotor is composed of IPM1 and IPM2 dual PWM current transformer, controlled by DSP1 and DSP2 respectively, and the communication is necessary to complete the ac excitation control strategy of variable speed constant frequency wind power generation. The rectifier or inverter state of side converter controlled by DSPZ is determined by the control signal $u_{d c}$. The main circuit is boost circuit by the operation of the rectifier, while the main circuit is bock circuit by the operation of the inverter, which causes a higher dc output voltage than an input $\mathrm{AC}$ voltage. Therefore, in the low dc output voltage situation, input side must adopt step-down transformer. The PC is used to realize the monitoring and management of control system, and responsible for receiving, real-time data display, or send data to the far side of the master control room. In addition, the PC also controls a dc machine under the laboratory condition to simulate the output characteristic of wind turbine, wet brake pipe governor in the picture is the execution of specific parts. To improve the quality of generator output power, power filtering device on the 
machine side also need to added, in order to reduce the harmonic pollution to power network.

\section{The experimental mill}

The experimental mill is shown in Fig .4. The wirewound induction generator is driven by a dc simulation wind machine. The frequency, amplitude and phase of the generator rotor is controlled by double PWM inverter to ensure the stator output voltage is consistent with the grid voltage, power grid, and the output power to the grid

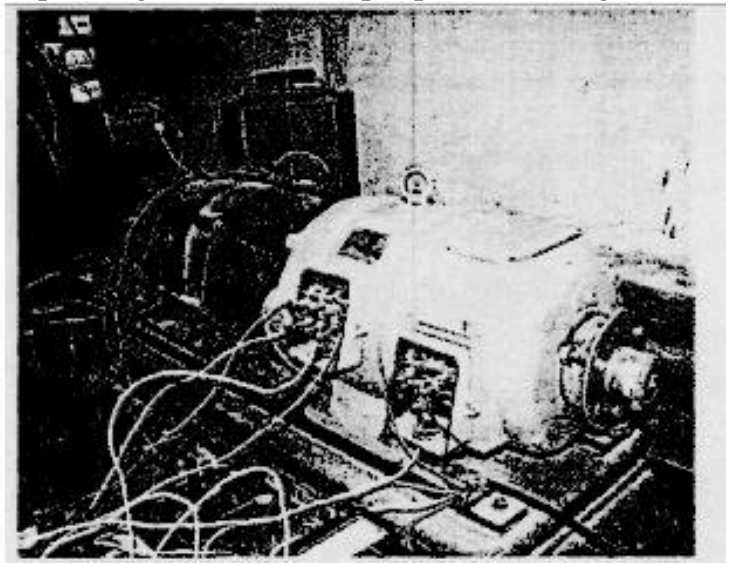

Figure 4. The diagram of experiment mill

2. The main loop of the ac excitation system

Dual PWM converter of side converter and the rotor side converter power device adopts intelligent power module (IPM), compared with the common IGBT module, the integrated drive and protective circuit makes the hardware circuit design and development of the system simple and reliable, improve the self-protection ability under the fault cases, also the system performance and reliability. Japanese Mitsubishi Electric Company PM50RSA120 intelligent power module is used in the device, whose withstand voltage is $1200 \mathrm{~V}$, rated current is $50 \mathrm{~A}$, and maximum switching frequency is $20 \mathrm{KHZ}$.

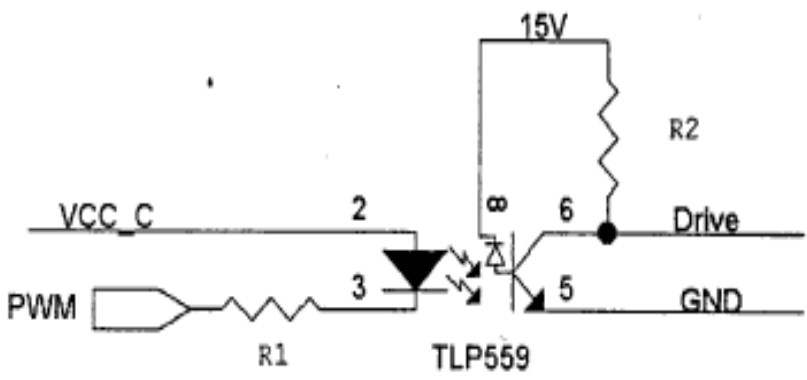

Figure 5. IPM drive isolation circuit

Seven IGBT tubes, drive circuit and protect circuit is integrated into IPM, the integration structure reduces the distribution of inductance by the introduction of line, and brings a better consistency opened and shut off of the device. Fig .5 exhibits the drive schematic diagram of IPM with bridge arm. The VCC - C is the controlled power source with protection function. PWM signals is the drive signal, and the Drive is the IPM Drive signal, the isolation adopts high-speed optical coupling TLP559, 15V and GND is the power supply to isolate system power supply. As the low level IPM Drive signal is effective, only for low level PWM signal, Drive is valid. This design can effectively prevent the mis-operation.

\section{DSP controller}

Due to the system to deal with large amount of data and real-time and accuracy requirement is high, we choose the United States Texas instruments digital signal processor (DSP) TMS320F240 and EVM entry plate as the controller, it is specially designed for motor digital control dedicated chip by $\mathrm{Tl}$ companies in the United States.

4. Signal detection circuit

Signal detection circuit can provide accurate information about the system run for control circuit, realize the system of decision-making and feedback control. This system involved in the voltage, current and photoelectric encoder signal detection. In voltage detection, in order to prevent the neutral drift caused by un-accurate phase voltage measurement, the system adopts a way of waste line voltage and calculation of phase voltage. In addition, no matter grid or motor, the current and voltage meet

$$
u_{a b}+u_{b c}+u_{c a}=0 \quad i_{a}+i_{b}+i_{c}=0
$$

So just need to check the line voltage $u_{a b}, u_{x}$ and line current $i_{a}, i_{b}$.

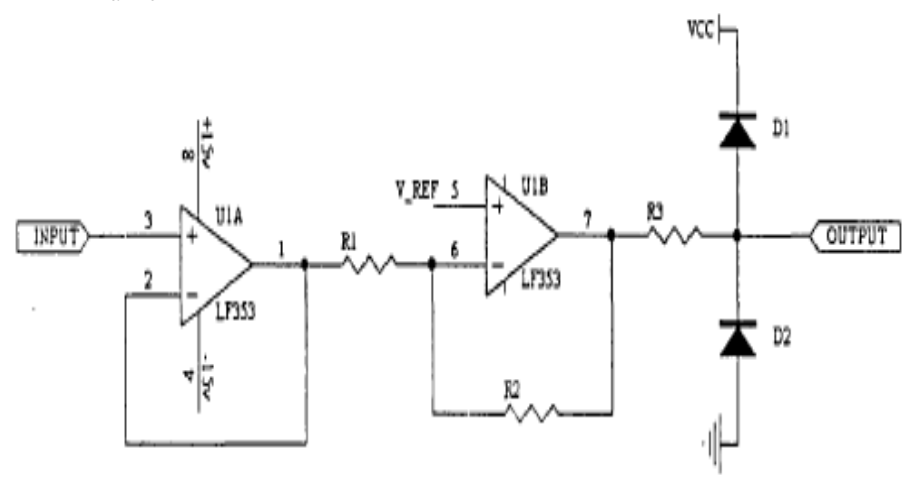

Figure 6. The circuit of signal following and level increasing

The detection of rotor speed and position signal is realized by a resolution of 1024 pulses/optical encoder which installated on the motor shaft. The encoder provides a positioning $\mathrm{z}$ pulse signal $\mathrm{A}$ and $\mathrm{B}$, the phase angle difference of each other is 90 . A and B signals can be sent to DSP event capture plastic mouth, capture unit count function are used to get the rotor speed and position.

Alternating current and voltage signal processing is relatively complicated, no matter hall element in current or voltage element, the output signals are all current mode, thus it must be converted into a voltage signal to be an acceptant $0 \sim 5 \mathrm{~V}$ level signal of DSP by the sampling resistor after adjusting proportion and increasing processing, then sent to DSP sampling by the hardware filtering. Following and level ascension circuit as shown in Fig .6: 


$$
\frac{u_{m}-V_{R E F}}{R_{1}}=\frac{V_{R E F}-u_{\text {out }}}{R_{2}}
$$

If $\mathrm{R}_{1}=\mathrm{R}_{2}, \mathrm{u}_{\text {out }}=2 \mathrm{~V}_{\mathrm{REF}}-\mathrm{u}_{\mathrm{m}}$

5. Remote grid control circuit

We choose dim lamps to test generator interconnection. When the generator stator voltage meets the grid-connection condition, a parallel grid control is carried out. At the same time, in the realization of IPM protection and the inverter and the rotor winding broken, it is also ensure that the stator winding is away from the grid and to design the remote grid control circuit, as shown in Fig .7.

Grid circuit is made up of three signal control relay contactor combinations contained manual grid button, software interconnection and hardware trouble-free. The parallel operation is achieved only when these three signals are high electricity level; and as long as there is a low level, the stator winding will take away from the grid. The system protection is realized by software interconnection enabling signal and hardware trouble-free signal of software and hardware.

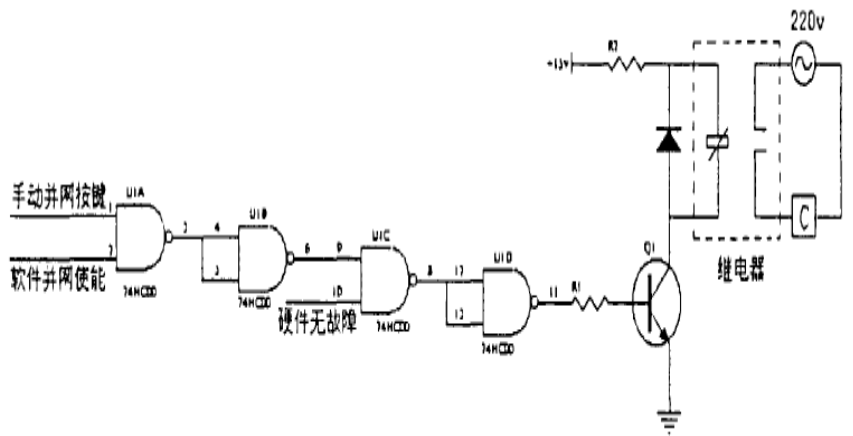

Figure 7. The remote grid control circuit

\section{Protection circuit}

As the experimental system will often work in the abnormal state of test, system protection function must be perfect. Frequency conversion device power device adopts the intelligent power module (IPM), which integrated the drive and protection circuit. The protection functions are as follows:

1) The control power supply under-voltage protection; 2) overheating protection; 3) over-current protection; 4) short circuit protection.

Overheating, over-current and short circuit protections are strong stress fault, which should be avoided repetitively.

The above several kinds of IPM self-protection for several IGBT module device is not unanimous action, and the output alarm signal is not stable; Just rely on the protective function of IPM itself may lead to system flow oscillation occurred, even damage the module is. Therefore, in order to ensure safe and reliable operation of the system, the protection of the auxiliary circuit must be added to form a complete multi-level protection system.

\section{CONCLUSION}

A variable speed constant frequency system was designed and implemented by implementing the unit, ac excitation system main circuit, DSP controller, signal detection circuit, remote grid control circuit and protection circuit.

\section{REFERENCES}

[1] A. Eid, H.El-Kishky, M. Abdel-Salam, M.T. El-Mohandes. On Power Quality of Variable-Speed Constant-Frequency Aircraft Electric Power Systems. Power Delivery,Vol. 25, 2010, PP. 55-65

[2] J.M.Alonso, M.S. Perdigão, D.G. Vaquero, A.J. H. R. Calleja, et al. Analysis, Design, and Experimentation on Constant-Frequency DC-DC Resonant Converters With Magnetic Control. Power Electronics,Vol. 27, 2012, PP. 1369-1382

[3] Y.Yang, K.Zhou, W. Lu. Robust repetitive control scheme for three-phase constant-voltage-constant-frequency pulsewidthmodulated inverters. Power Electronics,Vol. 5, 2012, PP. 669-677

[4] C. H. Chang ; F. Y. Wu ;Y. M. Chen. Modularized Bidirectional Grid-Connected Inverter With Constant-Frequency Asynchronous Sigma-Delta Modulation. Industrial Electronics, Vol. 59, 2012, PP 4088-4100

[5] L. Liu, Z. Dong. Study on PI Torque Controller of MW Variable Speed Constant Frequency Wind Turbine. Journal of Shanghai Electric Technology, Vol. 4, 2011, PP. 1-4

[6] J. Bao, W. Li, D. Zhang, Y. Lin, H. Liu. A Control Method for Inverse-pendulum Wave Power Generation System Based on Hydraulic Transmission to Maintain Pressure and Ensure Constant Frequency. Automation of Electric Power Systems, Vol. 37, 2013 PP. 18-22

[7] J. Biello, J.K. Hunter. Nonlinear Hamiltonian waves with constant frequency and surface waves on vorticity discontinuities. Communications on Pure and Applied Mathematics, Vol. 63, 2010, PP. 303-336

[8] C. A. Yeh, Y. S.Lai. Digital Pulsewidth Modulation Technique for a Synchronous Buck DC/DC Converter to Reduce Switching Frequency. Industrial Electronics, Vol. 59, 2012, PP. 550-561

[9] H. Zhu, Z. Jin, S. Hu, Y. Liu. Constant-frequency oscillation control for vibratory micro-machined gyroscopes. Sensors and Actuators A: Physical. Vol. 193, 2013, PP. 193-200

[10] F. Valenciaga. Second order sliding power control for a variable speed-constant frequency energy conversion system. Energy Conversion and Management, Vol. 51, 2010, PP. 3000-3008 